\title{
PLURIZENTRITITÄT DER DEUTSCHSPRACHIGEN TERMINOLOGIE IM AMTS- UND VERWALTUNGSBEREICH
}

\author{
Olena Kashchy ${ }^{1}$
}

\begin{abstract}
The article is devoted to the revealing and analysis of the lexical features of the Austrian version of the German language as a pluriscentric language in the field of management and administration. The lexical differences between the German and Austrian versions of the German language are so significant that they can hinder free orientation in the German-speaking space and correct language use. The existing lexical differences are partly caused by the social and public system peculiarities of the country, the specifics of the administrative structure and state broadcasting. The sources of the research are texts of Austrian media publications. Study material is consisted of lexical units obtained by continuous selection of Austrian print media "Standard" and "Österreich Spiegel". In the course of the study, in the texts of the printed publications were revealed lexical items, which are codified standard norms of the Austrian version of the German language. The analysis of available data allows us to classify several types of parallel forms in the Austrian and German variants of the German language: synonymous forms, denoting the same reality, but using different lexical items; synonymous lexical items to denote the same objects and phenomena, the distinction between which is purely formal; lexical items denoting realities, that are typical for only one country of the German-speaking space, or objects and phenomena whose similarities are limited; and lexical items that do not differ formally and are used in both versions of the pluricentric language, but have different meanings or synonyms only in one of the possible values.
\end{abstract}

\section{Keywords}

Pluristicency, variability, dominant variant, codified norm, parallel form, official and administrative language.

1. Einführung. Jeder Wandel in der Gesellschaft, sei es der technische Fortschritt, eine Wirtschaftskrise oder politische Entwicklungen, spiegelt sich in der Sprache und nämlich in der Lexik wieder. Das ist der Bereich, der sich am schnellsten und am auffälligsten verändert und aus diesem Grund immer das Interesse der Forscher weckt. Der Bereich der Lexik des modernen Deutsch ist trotz seiner Vielfalt am besten erforscht und in mehreren Wörterbüchern und Nachschlagewerken kodifiziert, was das Erlernen der Sprache und den praktischen Sprachgebrauch wesentlich erleichtern sollte. Als eine plurizentrische Sprache mit mehreren nationalen Zentren weist das Deutsche jedoch teils starke Unterschiede innerhalb dieser Varietäten auf. Dies gilt sowohl für die Bereiche der Grammatik, Pragmatik und Phonologie als auch für den Bereich der Lexik, wo diese Differenzen am deutlichsten gekennzeichnet sind. Die Existenz verschiedener standardsprachlicher Varietäten ist für die deutsche Sprache aus linguistischer Sicht unbestritten. Aus didaktischer Sicht hingegen aktuell ist die Frage, welches plurizentriche Wissen benötigen die Deutschlernenden, um im deutschsprachigen Raum zurechtzukommen, und ob und wie die aufgewiesenen Unterschiede im DaF-Unterrichtkonzept berücksichtigt werden müssen. In den Studien von Ulrich Ammon 2 und Rudolf Muhr 7 wurde der Begriff ,polyzentrisch“ oder „plurizentisch“ definiert, sowie auch die Merkmale einer plurizentrischen Sprache bezeichnet.

\footnotetext{
${ }^{1}$ Olena R. Kashchy, department of Foreign Languages № 2, National University "Odessa Law Akademy", 23 Fontanskaya Doroga, 65009, Ukraine; E-mail: elena.kashchy@gmail.com ORCID ID 0000000328758289
}

Die Plurizentrizität bedeutet, dass die Sprache mehrere nationale Zentren besitzt, jedes von denen eigene divergierende standartsprachliche Normen entwickelt, die in Wörterbüchern und Grammatiken kodifiziert sind. Diese kodifizierten Standardvarietäten weisen jedoch mehr Gemeinsamkeiten als Unterschiede innerhalb des gemeinsamen Sprachraums auf.

Laut Rudolf Muhr 9 zählt das Deutsche zu den plurizetrischen Sprachen: es ist eine offizielle Staatssprache bzw. Co- Staatssprache in drei souveränen Ländern, wo es uneingeschränkt gültig ist, d.h. die deutsche Sprache ist von drei nationalen Standardvarietäten vertreten, die über eigene kodifizierte sprachliche und kommunikative Normen verfügen.

Dieser Beitrag gibt den Überblick über lexikalische Merkmale der österreichischen Standardsprache innerhalb des Phänomens der Plurizentrik und ist das Ergebnis einer vergleichenden Analyse von zwei nationalen Standardvarietäten: der deutschen und der österreichischen.

Das Objekt der vorliegenden Untersuchung bilden die Texte von österreichischen Printmedien im Bereich der Staatsverwaltung. Der Gegenstand der Untersuchung sind lexikalische Einheiten, die für Standardvarietäten des Deutschen als geltende standardsprachliche Norm kodifiziert sind, aus kontrastiver Sicht. Die Untersuchung setzt sich zum Ziel, lexikalische Unterschiede zwischen der deutschen und der österreichischen Varietät des Deutschen als einer plurizentrischen Sprache im Bereich der Amts- und Verwaltungssprache festzustellen. Zu diesem Zweck werden folgende Aufgaben gelöst:

(1) Merkmale des Deutschen als einer plurizentrischen Sprache festgestellt; 
(2) die neuesten Studien der standardsprachlichen Varietäten des Deutschen analysiert;

(3) die lexikalischen Mittel, die in der Amts- und Verwaltungssprache als standardsprachliche Norm zweier Varietäten kodifiziert sind, verglichen;

(4) die im Bereich der Lexik aufgewiesenen Unterschiede zwischen der deutschen und der österreichischen Varietät der deutschen Sprache beschrieben und klassifiziert.

Die Hypothese der Untersuchung liegt darin, dass die standardsprachlichen Varietäten des Deutschen (in diesem Fall das „österreichische“ und das „deutsche“ Deutsch) wesentliche Unterschiede aufweisen, die aber nicht dialektal, sondern als eine geltende Norm betrachtet werden müssen. Trotz der überwiegenden Gemeinsamkeiten der Sprachvarietäten sind diese Unterschiede in der Amts- und Verwaltungssprache so beträchtlich, dass ihre Nichtberücksichtigung das Verständnis und das Zurechtfinden im deutschsprachigen Raum verhindern kann.

\section{Methoden}

2.1. Stichprobe. Die Forschungsmethoden richten sich nach dem Ziel, den Aufgaben und der Spezifika des Forschungsgegenstands. Für die Untersuchung werden die Methoden der linguistischen und der komparativen Analyse verwendet. Die Methode der linguistischen Analyse impliziert die wissenschaftliche und theoretische Analyse der Literatur sowie auch linguistische Interpretation der Ergebnisse. Die komparative Methode lässt sprachliche Elemente mehrerer Sprachen bzw. Sprachvarietäten vergleichen, in diesem Fall „österreichisches" und ,deutsches" Deutsch.

2.2. Material. Die Forschung basiert auf der Analyse von größten Printmedien Österreichs auf standardsprachliche Formen der nationalen Varietäten des Deutschen und ihre Verwendung bzw. Häufigkeit. Bei der Untersuchung stand der Vergleich der deutschen und der österreicheschenen Varietät im Vordergrund. Der lexikalische Bestand von Printmedien wurde genau gelesen und die darin verwendeten Austriazismen aufgelistet. Damit die Belege identifiziert und in die Bestandliste aufgenommen werden können, hat man parallel in den Nachschlagewerken - dem Österreichischen Wörterbuch und dem Duden - überprüft, ob die gefundenen Belege als Austriazismen ausgewiesen sind. Die bekanntesten und am besten erforschten lexikalischen Unterschiede sind in den Bereichen Küche, Gastronomie, Haushalstgegenstände und Möbelstücke 5 also in den Bereichen, die unmittelbar mit dem Alltagsleben der Sprecher zusammenhängen. Um festzustellen, wie beträchtlich die Unterschiede in anderen Lebensbereichen sind und wie oft sie in den Zeitungstexten vorkommen, wurden die österreichische Tageszeitung „Standard“" und die Zeitung „Österreich Spiegel“ zur Untersuchung herangezogen. Alle lexikalischen Einheiten, die für die österreichische Varietät des Deutschen als Standard angesehen werden können, wurden nach Rubriken klassifiziert und in die Tabelle aufgenommen.
Gleichzeitig werden die Bedeutung dieser Wörter und ihre parallele Form im deutschen Deutsch sowie auch das Vorkommen dieser lexikalischen Einheiten in beiden Nachschlagewerken angeführt.

3. Resultate und Diskussion. In diesem Beitrag geht man von der Plurizentritität der deutschen Sprache aus. Aus diesem Grund sind zuerst der Begriff und die Merkmale einer plurizentrischen Sprache zu definieren. Allen plurizenrischen Sprachen ist es gemein, dass sie über mehrere Standardvarianten verfügen und dass die sprachlichen Merkmale jeder Variante kodifiziert sind. Jede plurizentrische Sprache muss folgenden Kriterien entsprechen:

- sie kommt in mehreren souveränen Staaten vor

- sie ist in diesen Staaten offiziell anerkannt und erfüllt die Funktion der Staats- und Amtsprache bzw. einer der Staatsprachen oder Minderheitssprache.

- die Sprecher einer Varietät der Gesamtsprache halten diese Sprache nicht für eigenständig, sondern für einen Teil der Gesamtsprache 1.

Sprachliche Normen der Standardvarietäten einer plurizentrischen Sprache sind in Wörterbüchern, Nachschlagewerken und Grammatiken kodifiziert. Jede staatliche Varietät ist geltende Norm nicht nur in Behörden, Verwaltung und Gesetzgebung, sondern auch in den Bildungseinrichtungen. Die aufgewiesenen Unterschiede sind durch unterschiedliche Lebensbedingungen, Besonderheiten der sozialen Verhältnisse, sowie auch durch die Identifizierung der Muttersprachler verschiedener nationaler Standardvarietäten bedingt, die sich in der Sprache wiederspiegelt.

$\mathrm{Zu}$ betonen ist, dass trotz der Varietät der Sprachnormen und linguistischen Unterschiede auf der morphologischen, semantischen, pragmatischen und phonologischen Ebene, ist jede plurizentrische Sprache eine Gesamtsprache, und seinen nationale Variante weist mehr Gemeinsamkeiten auf. Für jede plurizentrische Sprache ist eine gewisse Asymmetrie kennzeichnend: eine der nationalen Varietäten ist meist dominant und wird als solche von Sprechern anderer nationaler Varietäten wahrgenommen; die sprachlichen Formen der dominanten Variante werden von ihnen als bessere und schönere bewertet. Die Sprecher der dominanten Varietät hingegen tendieren dazu, andere Sprachformen mit den regionalen Dialekten ihres Landes gleichzusetzen.

Was die deutsche Sprache betrifft, ist solche dominante Varietät definitiv das „deutsche“ Deutsch, was durch die große Sprecheranzahl, wirtschaftliche Stärke und politische Bedeutung Deutschlands zu erklären ist. Dies führt dazu, dass sich die Sprecher anderer nationaler Varietäten an den Normen der dominierenden Sprache orientieren, während sie über die Normen der Standardsprache ihres Staates, sei es das österreichische oder das schweizerische Deutsch, sowie auch über ihre sprachliche Relevanz in bestimmten kommunikativen Situationen unsicher sind. Die Dominanz von sprachlichen Formen des 
„deutschen“ Deutsch im Vergleich zu dem „österreichischen“ oder dem „schweizerischen“ zeigt sich in erster Linie in Schrift, wo die meisten Entlehnungen aus dem lexikalischen Bestand des „deutschen“ Deutsch auftreten und wo man sich an den grammatischen Normen gerade dieser Varietät hält. Gleichzeitig sind das österreichische und das schweizerische Deutsch kodifizierte Standardsprachen und sollen nicht als ein Dialekt, als eine Abweichung von der allgemein geltenden Norm wahrgenommen werden, sie sind gleichzustellen.

Laut der Definition von Rudolf Muhr 9 ist das „österreichische" Deutsch:

- eine nationale Varietät des Deutschen;

- die Gesamtheit aller sprachlichen und kommunikativen Formen des Deutschen in der österreichischen Republik;

- alle regionalen und dialektalen Formen des Deutschen in Österreich;

- der für die Sprecher charakteristische Umgang mit der Sprache und ihren Varianten.

Das österreichische Deutsch als eine Standardvarietät der plurizentrischen Sprache besitzt seine eigenen linguistischen Normen. Diesen Normen kann man im DaF-Unterricht nur bedingt gerecht werden. Es stellt sich die Frage, inwieweit das plurizentrische Prinzip sowie auch die Berücksichtigung von phonetischen, morphologischen, grammatischen und pragmatischen Normen der Standardvarietäten des Deutschen für den DaF- Unterricht und somit für die Deutschlernenden relevant sind. In modernen Lehrbüchern und Lehrwerken wird die plurizentrische Sprachrealität nur bedingt berücksichtigt und die auftretenden lexikalischen Einheiten und grammatischen Formen werden nicht als geltende Norm, sondern eher als eine Abweichung von der Standardsprache, als dialektal oder umgangssprachlich betrachtet, was der Sprache zusätzliche emotionale Konnotation verleiht. 8 . Dass sich die DaF-Lehrwerke der sprachlichen Realität nicht annähern und die wichtigsten Merkmale der nationalen Varietäten nicht vermitteln ist die Folge der Asymmetrie. Gleichzeitig sind die Unterschiede beträchtlich hoch und decken alle Sprachebenen ab. Die meisten zeigen sich im phonetischen und lexikalischen Bereich, sowohl in der mündlichen als auch in der schriftlichen Sprache. Oft wird angenommen, dass die meisten Unterschiede in den Bereichen Gastronomie und Haushaltsgegenstände auftreten und können auf solche Weise die Kommunikation im Beruf, in der Bildung oder in der Verwaltung kaum verhindern. Aus der Untersuchung der Printmediatexte ergibt sich aber, dass die lexikalischen Einheiten im Bereich der Staatsverwaltung wesentliche Unterschiede aufweisen und die Häufigkeit ihres Gebrauchs und die Kodifizierung in den Wörterbüchern und Machschlagewerken lässt sie als geltende Norm der Sprachvarietäten bewerten. Die Ergebnisse der durchgeführten Analyse sind in der Tabelle angeführt (Anhang A).

Aus der Tabelle ergibt sich, dass das Vorkommen von lexikalischen Einheiten, die gerade für die öster- reichische Varietät des Deutschen als Standard angesehen werden, die Häufigkeiten ihrer Verwendung sowie auch ihre Präferenz gegenüber den Formen der "deutschen" Varietät in Printmedientexten, gerade sie als kodifizierte Standardnormen bezeichnen lassen. Die aufgewiesenen lexikalischen Unterschiede im Bereich der Lexik sind beträchtlich, obwohl die Sprecher beliebiger Standardvarietät über die passive Sprachkompetenz verfügen, die sie die Bedeutung der Wörter verstehen lässt. Die Übersetzung solcher lexikalischen Einheiten von einem Nicht-Muttersprachler sowie auch ihr Verständnis beim Spracherlernen können hingegen wesentliche Schwierigkeiten bereiten. Wenn es um lexikalische Einheiten geht, ist es relevant, auf die Klassifikation von Rudolf Muhr einzugehen. Laut Rudolf Muhr 5 lassen sich in der Lexik drei Grundkategorien unterscheiden: Parallelformen, Teilsynonyme und Polysynonyme sowie auch Präferenzunterschiede innerhalb eines lexikalischen Sprachfeldes. Die Analyse der aufgelisteten Belege lässt die Parallelformen in drei Typen einteilen. Im ersten Fall handelt es sich um synonyme Wörter, die dieselbe Sache oder denselben Begriff bezeichnen, d.h. die Sache existiert in beiden Staaten, kommt aber durch formal unterschiedliche lexikalische Einheiten zum Ausdruck. Zum Beispiel:

Tabelle 2

Echte binationale Parallelformen

\begin{tabular}{|l|l|}
\hline Österreichisches Deutsch & \multicolumn{1}{|c|}{ Deutsches Deutsch } \\
\hline Familienbeihilfe & Familiengeld \\
\hline Bundesheer & Bundeswehr \\
\hline Landeshauptmann & Ministerpräsident \\
\hline Pickerl & TÜV-Plakete \\
\hline Hackler & Arbeiter \\
\hline Erlag & Beitrag \\
\hline deppert & dämlich \\
\hline
\end{tabular}

Solche Parallelformen können konnotative oder stilistische Unterschiede aufweisen.

In manchen Fällen sind formale Unterschiede zwischen den lexikalischen Einheiten, die denselben Begriff bezeichnen, nur geringfügig und die Ausdrücke sehr ähnlich sind. Gerade solche Parallelformen werden oft als unkorrekte, fehlerhafte angesehen.

Geringfügig verscheidende Parallelformen

\begin{tabular}{|l|l|}
\hline Österreichisches Deutsch & \multicolumn{1}{|c|}{ Deutsches Deutsch } \\
\hline Anbot & Angebot \\
\hline anbandeln & anbändeln \\
\hline Dienstgeber & Arbeitgeber \\
\hline Meldezettel & Anmeldezettel \\
\hline Pensionkassa & Pensionkasse \\
\hline Werber & Bewerber \\
\hline marod & marode \\
\hline Jus & Jura \\
\hline
\end{tabular}


In Printmedien kommen lexikalische Einheiten vor, die die Begriffe bezeichnen, die nur für ein Land typisch sind, sowie auch Gegenstände und Erscheinungen, deren Ähnlichkeit bedingt ist. In diesem Fall ist es schwierig eine echte Parallelform zu finden, weil es um die Gegenstände und Einrichtungen geht, die es nur in einem Land gibt oder die nur bedingt ähnlich sind. In diesem Fall ist es schwierig die genaue Entsprechung zu finden, weil der Inhalt damit zu ungenau bezeichnet wird.

Tabelle 4

Unechte Parallelformen-Sachspezifika.

\begin{tabular}{|l|l|l|}
\hline $\begin{array}{c}\text { Österreichi- } \\
\text { sches Deutsch }\end{array}$ & \multicolumn{1}{|c|}{ Значение } & $\begin{array}{l}\text { Deutsches } \\
\text { Deutsch }\end{array}$ \\
\hline Trafik & $\begin{array}{l}\text { eine Verkaufsstelle, } \\
\text { wo in ersten Linie } \\
\text { Tabakwaren aber auch } \\
\text { Printmedien verkauft } \\
\text { werden }\end{array}$ & $\begin{array}{l}\text { Kiosk/ } \\
\text { Tabakladen }\end{array}$ \\
\hline perlustrieren & $\begin{array}{l}\text { jemanden aufhalten, } \\
\text { überprüfen und durch- } \\
\text { suchen }\end{array}$ & $\begin{array}{l}\text { aufhalten/ } \\
\text { befragen/ } \\
\text { durchsuchen }\end{array}$ \\
\hline Hacklerregelung & & - \\
\hline
\end{tabular}

In diesem Fall vermitteln die lexikalischen Einheiten der ,deutschen” Varietät nur teilweise die Bedeutung des Wortes der „österreichischen” oder es gibt gar keine Entsprechung in dem „deutschen“ Deutsch. Dies ist auf unterschiedliche Lebens- und soziale Bedingungen in Deutschland und Österreich zurückzuführen.

Anhand der Analyse der gefundenen Belege lässt sich eine weitere Gruppe unterscheiden: lexikalische Einheiten, die sich formal nicht oder kaum unterscheiden und in beiden Sprachvarietäten im Gebrauch sind, jedoch unterschiedliche Bedeutungen haben.

Tabelle 5

\begin{tabular}{|c|c|c|c|}
\hline 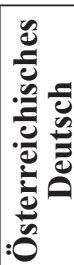 & ف를 & 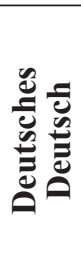 & 窇 \\
\hline 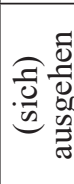 & ausreichen, genügen & $\begin{array}{l}\bar{\Xi} \\
\bar{\Xi} \\
\mathbb{8} \\
0 \\
\bar{\sigma}\end{array}$ & $\begin{array}{l}\text { mit einer bestimmten } \\
\text { Absicht das Haus } \\
\text { verlassen, zum } \\
\text { Vergnügen ein Lokal } \\
\text { o.Ä. aufsuchen }\end{array}$ \\
\hline 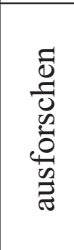 & $\begin{array}{l}\text { 1. jemanden } \\
\text { polizeilich ausfindig } \\
\text { machen } \\
\text { 2. etwas sorgfältig } \\
\text { erforschen und } \\
\text { jemanden eingehend } \\
\text { befragen }\end{array}$ & 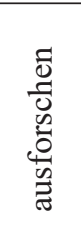 & $\begin{array}{l}\text { nur in der Bedeutung } \\
\text { jemanden befragen } \\
\text { oder auch etwas } \\
\text { erforschen }\end{array}$ \\
\hline
\end{tabular}

Perspektiven: Aus der Analyse ergibt sich, dass formal identische lexikalische Einheiten einer polyzentrischen Sprache in ihren Standardvarietäten unterschiedliche Bedeutungen haben können oder nur in einer der möglichen Bedeutungen synonym sind.

Außerdem weisen nationale Varietäten einer polyzentrischen Sprache lexikalische Unterschiede auf, die mit den Präferenzen der Sprecher beim Gebrauch bestimmter lexikalischer Einheiten zusammenhängen; dass heißt, wenn es in beiden Sprachvarietäten mehrere synonyme Formen wird, wird eine davon bevorzugt. Zum Beispiel von zwei synonymen Varianten: "sehen“ oder „schauen“ wird von einem Österreicher die zweite Variante bevorzugt, dasselbe gilt für die Wörter Geldbörse und Geldbeutel / Geldtasche, wobei der Begriff Geldbörse typisch für "österreichisches" Deutsch ist, wenn auch beide anderen Varianten kodifizierte Normen der Standardsprache sind.

Aus der Analyse von österreichischen Printmediatexten und der sprachlichen Situationen, in denen die lexikalischen Unterschiede zwischen der österreichischen und der deutschen Varietät der deutschen Sprache am häufigsten vorkommen, ergibt sich, dass gerade die Printmediasprache, die Amts- und Verwaltungssprache einen der Bereiche bilden, in denen die Plurizentrizität des Deutschen am deutlichsten zum Ausdruck kommt. Der plurizentrische Sprachgebrauch zeigt sich also in allen Bereichen, die mit den Besonderheiten des gesellschaftlichen und sozialen Lebens sowie auch mit der Verwaltungsstruktur Österreichs zusammenhängen.

Das Deutsche ist also eine plurizentrische Sprache mit drei gleichwertigen Varietäten. Lexikalische Unterschiede, die die sprachlichen Varietäten (in diesem Fall die deutsche und die österreichische) aufweisen, sind so beträchtlich, dass ihr Unkenntnis verhindern kann, sich im deutschsprachigen Raum zurechtzukommen. Die Muttersprachler verfügen in der Regel über passive Sprachkompetenz, die die Rezeption einer anderen Varietät erleichtert. Den Deutschlernenden hingegen kann die Plurizentrizität der Sprache Schwierigkeit bereiten. Ebenso sind die lexikalischen Unterschiede einzelner Sprachvarietäten beim Übersetzen zu berücksichtigen, wo es sich nicht nur um den korrekten, sondern auch um den bewussten und sozial relevanten Sprachgebrauch handelt. Der plurizentrische Ansatz ist auch für den DaF Unterricht von großer Bedeutung. Der DaF-Unterricht soll durch die Nähe an der sprachlichen und kommunikativen Realität des Deutschen gekennzeichnet sein, das heißt die kommunikativen und linguistischen Spezifika von deutschsprachigen Ländern berücksichtigen. 
Anhang A.

Tabelle 1

Vorkommen von Parallelformen in Standardvarietäten des Deutschen

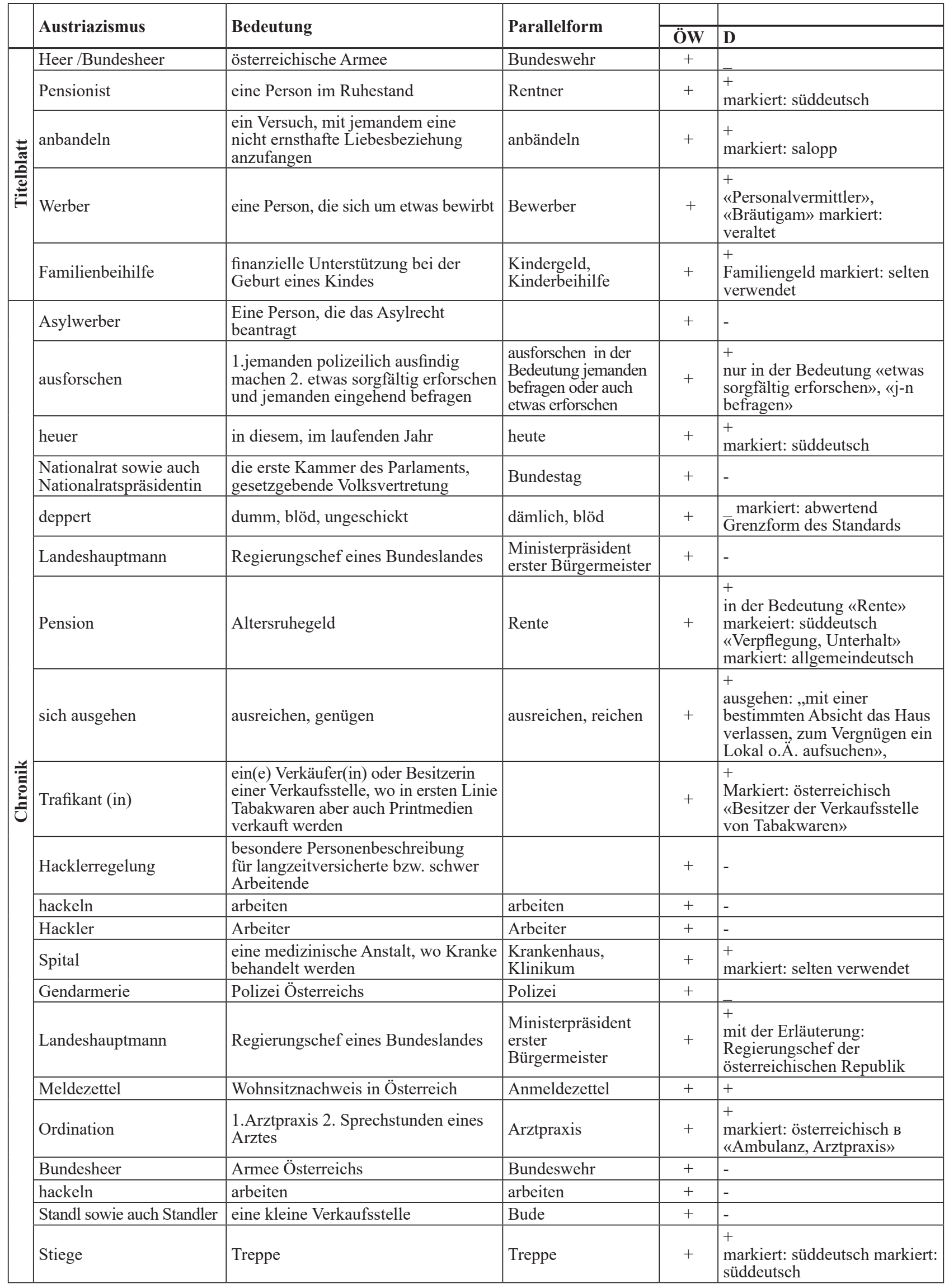


Ende der Tabelle 1

\begin{tabular}{|c|c|c|c|c|c|}
\hline & \multirow{2}{*}{ Austriazismus } & \multirow{2}{*}{ Bedeutung } & \multirow{2}{*}{ Parallelform } & & \\
\hline & & & & ÖW & $\mathbf{D}$ \\
\hline \multirow{10}{*}{ : } & Amtstag & Sprechstunden bei einer Behörde & Sprechtag & + & - \\
\hline & Börsl & Verkleinerung von Geldbörse & $\begin{array}{l}\text { Geldbeutel } \\
\text { Geldtasche } \\
\text { Pormonnaie }\end{array}$ & + & - \\
\hline & heuer & in diesem, im laufenden Jahr & heuer & + & $\begin{array}{l}+ \\
\text { markiert: süddeutsch }\end{array}$ \\
\hline & Justizanstalt & $\begin{array}{l}\text { Justizvollzugsanstalt, } \\
\text { Gefangenenhaus, } \\
\text { Polizeianhaltezentrum }\end{array}$ & Justizvollzugsanstalt & + & 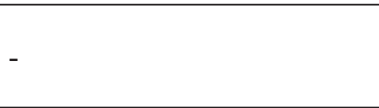 \\
\hline & Spital & $\begin{array}{l}\text { medizinische Anstalt, wo Kranke } \\
\text { behandelt werden }\end{array}$ & \begin{tabular}{|l|} 
Krankenhaus, \\
Klinikum
\end{tabular} & + & + \\
\hline & Unterstand & $\begin{array}{l}\text { eine Stelle, wo man sich zum Beispiel } \\
\text { zum Schutz von Regen; Schnee oder } \\
\text { Gefahr unterstellen kann }\end{array}$ & Unterkunft & + & $\begin{array}{l}+ \\
\text { markiert:militär «Unterstand, } \\
\text { Bunker » }\end{array}$ \\
\hline & perlustrieren & $\begin{array}{l}\text { jemanden aufhalten, überprüfen und } \\
\text { durchsuchen }\end{array}$ & & + & $\begin{array}{l}+ \\
\text { markiert: österreichisch } \\
\text { jemanden aufhalten, um die } \\
\text { Dokumenten zu überprüfen } \\
\end{array}$ \\
\hline & marod & $\begin{array}{l}\text { sich nicht wohl fühlen, auf dem Weg } \\
\text { zum Verfallen sein (nicht nur in } \\
\text { Bezug auf eine Person) }\end{array}$ & marode & + & $\begin{array}{l}+ \\
\text { markiert: österreichisch . }\end{array}$ \\
\hline & juridisch & rechtlich & juristisch & + & - \\
\hline & \begin{tabular}{|l|} 
Jus \\
\end{tabular} & Rechtswissenschaft & Recht, Jura & & \\
\hline \multirow{5}{*}{ : } & Zivildiener & $\begin{array}{l}\text { eine Person, die den Zivildienst } \\
\text { leistet }\end{array}$ & & + & - \\
\hline & \begin{tabular}{|l|} 
anpatzen \\
\end{tabular} & besudeln, bekleckern & bekleckern & + & - \\
\hline & Dienstgeber & $\begin{array}{l}\text { eine Person, die den Leuten } \\
\text { Arbeitsplätze anbietet, die sie fest } \\
\text { anstellen kann }\end{array}$ & Arbeitgeber & + & + \\
\hline & Erlag & & Zahlung, Beitrag & + & $\begin{array}{l}+ \\
\text { markiert: österreichisch } \\
\end{array}$ \\
\hline & Pfusch & Schwarzarbeit & Schwarzarbeit & + & $\begin{array}{l}+ \\
\text { markiert: umgangssprachlich }\end{array}$ \\
\hline \multirow{13}{*}{ 焉 } & Hacklerregelung & $\begin{array}{l}\text { besondere Personenbeschreibung } \\
\text { für langzeitversicherte bzw. schwer } \\
\text { Arbeitende }\end{array}$ & & + & - \\
\hline & Arbeiterkammer & $\begin{array}{l}\text { Kammer für Arbeiter und Angestellte, } \\
\text { gesetzliche Vertretung der } \\
\text { Arbeitnehmer }\end{array}$ & & + & markiert: österreichisch \\
\hline & heuer & in diesem, im laufenden Jahr & heute & + & $\begin{array}{l}+ \\
\text { markiert: süddeutsch }\end{array}$ \\
\hline & Landeshauptmann & Regierungschef eines Bundeslandes & $\begin{array}{l}\text { Ministerpräsident } \\
\text { erster Bürgermeister }\end{array}$ & + & $\begin{array}{l}+ \\
\text { mit der Erläuterung: } \\
\text { Regierungschef der } \\
\text { österreichischen Republik }\end{array}$ \\
\hline & Spital & $\begin{array}{l}\text { medizinische Anstalt, wo Kranke } \\
\text { behandelt werden }\end{array}$ & $\begin{array}{l}\text { Krankenhaus, } \\
\text { Klinikum }\end{array}$ & + & + \\
\hline & \begin{tabular}{|l|} 
Pensionkassa \\
\end{tabular} & & Pensionkasse & + & - \\
\hline & \begin{tabular}{|l|} 
abhausen \\
\end{tabular} & Pleite machen & bankrott gehen & + & - \\
\hline & Anbot & & Angebot & + & - \\
\hline & befürsorgen & aufpassen, pflegen, sich sorgen & betreuen & + & + \\
\hline & Freunderlwirtschaft & $\begin{array}{l}\text { Dienstversäumnis zugunsten von } \\
\text { Verwandten und Bekannten }\end{array}$ & Vetternwirtschaft & + & - \\
\hline & Körbergeld & & Provision & + & - \\
\hline & grauslich & ekelhaft, hässlich, unangenehm & gräuslich & + & + $+{ }_{\text {markiert: süddeutsch }}$ \\
\hline & Pickerl & Begutachtungplakette, Vignette & TÜV-Plakette & + & - \\
\hline
\end{tabular}




\section{LITERATURVERZEICHNIS}

Ammon Ulrich. Die deutsche Sprache in Deutschland, Österreich und in der Schweiz / Ammon Ulrich // Der Gruyter. 1995. - C. 13-27.

Ammon Ulrich. Vorschläge zur Typologie nationaler Zentren und nationaler Varianten bei plurinationalen Sprachen / ÖBV. 1995. - C. 112-147.

Claluna Monika. Alles unter einem D-A-CH-L? Oder: Wie viel Plurizentrik verträgt ein Lehrwerk/Claluna Monika//Fremdsprache Deutsch. - 2007. - C. 37-68.

Hägi, Sara: Bitte mit Sahne / Rahm / Schlag: Plurizentrik im Deutschunterricht. In: Fremdsprache Deutsch (2007) H. 37 , S. 5-13.

Ebner Jacob. Wie sagt man in Österreich. Wörterbuch des österreichischen Deutsch 3 / Ebner Jacob // Dudenverlag. - 2008. -

C. 109-43.

Elspaß, Stephan: Zwischen „Wagen“ und „Wägen“ abwägen. Sprachvariation und Sprachvarietätenpolitik im Deutschunterricht.

In: Fremdsprache Deutsch (2007) H. 37, S. 30-36.

Muhr Rudolf. Das Deutsche als plurizentrische Sprache und Didaktik des Deutschen als plurizentrischer Sprache / Muhr Rudolf // ÖBV. - 1995. - C. 112-151.

Muhr Rudolf. Die linguistischen Merkmale der nationalen Varietät des Deutschen im Überblick / Muhr Rudolf // ÖBV. - 2000. C. $37-80$.

Muhr Rudolf. Grammatische und pragmatische Merkmale des österreichischen Deutschen / Muhr Rudolf, Schrodt Richard, Wiesinger Peter // ÖBV. - 1995. - C. 208-234.

Muhr, Rudolf: Das Österreichische Deutsch in Linguistik und Sprachunterricht seit 1945. Ein Bericht. In: Der Ginkobaum. Germanistisches Jahrbuch für Nordeuropa. Helsinki 1996, S. 220-238.

Pusswald Christina.Das plurizentrische Modell in der Plurizentrik des DaF-Unterrichts:eine Analyse anhand der österreichischen Deutsch/Pusswald Christina//Graz, Uniiv, Diss. - 2007.

Sedlaczek, Robert: Das österreichische Deutsch: wie wir uns von unserem großen Nachbarn unterscheiden. Wien: Ueberreuter 2004.

Kurnik, Jutta Elisabeth: Österreichisches Deutsch und seine nachbarsprachlichen Bezüge: die Lexik der Alltagssprache Österreichs und Bayerns im Vergleich. Eine empirische Studie. Graz, Univ., Dipl.-Arb. 1998.

\section{WÖRTERBÜCHER}

Langenscheidts Großwörterbuch Deutsch als Fremdsprache. Das neue einsprachige Wörterbuch für Deutschlernende. Hrsg. von Dieter Götz, Günther Haensch und Hans Wellmann. Berlin [u.a.]: Langenscheidt 1993.

Pons Basiswörterbuch Deutsch als Fremdsprache. Das einsprachige Lernerwörterbuch zum neuen Zertifikat Deutsch. Bearbeitet von Dörthe Hecht und Annette Schmollinger. Vollständige Neuentwicklung 1999. Stuttgart: Klett International 1999.

Variantenwörterbuch des Deutschen. Die Standardsprache in Österreich, der Schweiz und Deutschland sowie in Liechtenstein, Luxemburg, Ostbelgien und Südtirol. Hrsg. von Ulrich Ammon [u.a.] Unter Mitarbeit von Rhea Kyvelos [u.a.] Berlin, New York: de Gruyter 2004.

\section{REFERENZEN}

Ammon, U. (1995) Die deutsche Sprache in Deutschland, Österreich und in der Schweiz, Der Gruyter.

Ammon U. (1995) Vorschläge zur Typologie nationaler Zentren und nationaler Varianten bei plurinationalen Sprachen. ÖBV.

Claluna M. (2007) Alles unter einem D-A-CH-L? Oder: Wie viel Plurizentrik verträgt ein Lehrwerk / Claluna Monika, Fremdsprache Deutsch.

Hägi, S. (2007) Bitte mit Sahne/Rahm/Schlag: Plurizentrik im Deutschunterricht, Fremdsprache Deutsch.

Ebner J. (2008) Wie sagt man in Österreich. Wörterbuch des österreichischen Deutsch 3 / Ebner Jacob,Dudenverlag.

Elspaß, St. (1995) Zwischen „Wagen“ und „Wägen“ abwägen. Sprachvariation und Sprachvarietätenpolitik im Deutschunterricht. In: Fremdsprache Deutsch.

Muhr R. (1995) Das Deutsche als plurizentrische Sprache und Didaktik des Deutschen als plurizentrischer Sprache / Muhr Rudolf, ÖBV.

Muhr R. (2000) Die linguistischen Merkmale der nationalen Varietät des Deutschen im Überblick/Muhr Rudolf. ÖBV, pp 37-80.

Muhr R (1995). Grammatische und pragmatische Merkmale des österreichischen Deutschen/Muhr Rudolf, Schrodt Richard, Wiesinger Peter. ÖBV, pp. 208-234.

Pusswald Chr. (2007) Das plurizentrische Modell in der Plurizentrik des DaF-Unterrichts:eine Analyse anhand der österreichischen Deutsch/Pusswald Christina, Graz, Uniiv, Diss.

Sedlaczek R. (2004) Das österreichische Deutsch: wie wir uns von unserem großen Nachbarn unterscheiden. Wien: Ueberreuter.

Kurnik, J. E. (1998) Österreichisches Deutsch und seine nachbarsprachlichen Bezüge: die Lexik der Alltagssprache Österreichs und Bayerns im Vergleich. Eine empirische Studie. Graz, Univ., Dipl.-Arb.

\section{Анотація}

Стаття присвячена виявленню та аналізу лексичних особливостей австрійського варіанту німецької мови як мови плюрицентричної в галузі управління та адміністрування. Лексичні відмінності німецького та австрійського варіанту німецької мови $є$ настільки істотними, що можуть перешкоджати вільній орієнтації у німецькомовному просторі та коректному використанню мови. Наявні лексичні відмінності частково обумовлені особливостями соціального та суспільного устрою країни, специфікою Ії адміністративної структури та державного мовлення. Джерелом дослідження є тексти австрійських 
друкованих видань засобів масової інформації. Матеріал дослідження склали лексичні одиниці, отримані шляхом суцільного добору з австрійських друкованих медіа Standard та Österreich Spiegel. В ході дослідження в текстах друкованих видань виявлені лексичні одиниці, що є кодифікованими стандартними нормами австрійського варіанту німецької мови. Аналіз наявних даних дозволяє класифікувати декілька видів паралельних форм у австрійському та німецькому варіантах німецької мови: синонімічні форми, що позначають одну й ту саму реалію, але за допомогою різних лексичних одиниць; синонімічні лексичні одиниці для позначення однакових предметів та явищ, відмінність між якими $є$ суто формальною; лексичні одиниці, що позначають реалії, типові тільки для однієї країни німецькомовного простору, або предмети та явища, схожість між якими є обмеженою; та лексичні одиниці, що не відрізняються формально та використовуються в обох варіантах плюрицентричної мови, але мають різні значення або синонімічні лише у одному з можливих значень.

\section{Ключові слова}

Плюрицентричність, варіативність, домінантний варіант, кодифікована норма, паралельна форма, офіційна та адміністративна мова. 